\title{
À PROPOS DE LA SIGNIFICATION DES DÉPENDANCES ENTRE CRITÈRES : QUELLE PLACE ET QUELS MODES DE PRISE EN COMPTE POUR L'AIDE À LA DÉCISION?*
}

\author{
BERNARD ROY ${ }^{1}$
}

\begin{abstract}
Résumé. Soit $F$ une famille de critères conçue pour asseoir un modèle de préférences global sur un ensemble $A$ d'actions potentielles (ou alternatives). On se place ici dans une perspective d'aide à la décision et dans l'hypothèse où des dépendances (encore appelées interactions) sont susceptibles d'exister entre certains des critères de $F$. On commence (cf. Sect. 2.1) par préciser ce que signifie l'affirmation «il existe des dépendances entre certains des critères de $F \gg$ (Déf. 1). On s'intéresse ensuite à deux formes classiques de dépendances ( $c f$. Sect. 2.2). Dans la Section 3, on cherche à apporter des éléments de réponse à la question : comment mettre en évidence les dépendances s'il en existe et comment les rendre intelligibles pour les parties prenantes au processus de décision? On définit trois types de situations particulièrement dignes d'intérêt car aisément intelligibles et faciles à cerner. Dans la section suivante, on examine les possibilités qu'offrent un certain nombre de modèles d'agrégation pour prendre en compte explicitement des dépendances. On met notamment en évidence ( $c f$. Sect. 4.2) les possibilités et limites de l'intégrale de Choquet. Tout au long de l'article, des exemples sont introduits pour illustrer les propos.
\end{abstract}

Mots Clés. Dépendences entre critères, modélisation des préférences, procédures internactives, intégrale de Choquet, méthodes ELECTRE.

Received October 4, 2006. Accepted February 14, 2009.

* Ce sujet a fait l'objet d'une communication au `e congrès de la Société Française de Recherche Opérationnelle et d'Aide à la Décision, Lille, France, 6-8, février 2006.

1 LAMSADE, Université Paris-Dauphine, Place du Maréchal De Lattre de Tassigny, 75775 Paris Cedex, France; roy@lamsade.dauphine.fr 


\begin{abstract}
About the meaning of dependencies between criteria meaning: how to take them into account for decision aiding? Let $F$ be a family of criteria built for assessing a comprehensive preference model on a set $A$ of actions (or alternatives). Let us put ourselves in a decision aiding perspective, and with the hypothesis where dependencies can exist between some criteria of $F$. We start (see Sect. 2.1) by specifying what the assertion "there exist dependencies between some criteria of $F$ " means (Def. 1). Then we consider two usual forms of dependencies (see Sect. 2.2). In Section 3, we attempt to give basic principles to answer the question: how to put into light dependencies if there exist some, and how to make them intelligible for stakeholders in a decision process? We define three types of situations particularly worthy of interest, because they are clearly intelligible and easy to surround. In the next section, we examine the possibilities provided by a certain number of aggregation models for taking explicitly into account dependencies. We particularly bring to the fore the possibilities and limits of Choquet integral (see Sect. 4.2). All along the paper, examples are introduced in order to illustrate the intention.
\end{abstract}

Keywords. Dependencies between criteria, preference modelling, interactive procedures, Choquet integral, ELECTRE methods.

Classification Mathématique. 90XX.

\title{
1. INTRODUCTION
}

On se place ici dans une perspective d'aide à la décision. On considère une famille $F$ de $n(\geqslant 2)$ critères $g_{1}, \ldots, g_{n}$ conçue pour asseoir un modèle de préférences global sur un ensemble $A$ (non nécessairement fixé a priori) d'actions potentielles (ou alternatives). Dans ces conditions, si des dépendances sont susceptibles d'exister entre certains des critères de $F$, il me paraît nécessaire, pour construire le modèle de préférences global, de chercher à répondre aux questions suivantes :

(1) De quelles formes de dépendances s'agit-il?

(2) Comment, si l'on suppose qu'il existe des dépendances, les mettre en évidence et les rendre intelligibles pour les parties prenantes dans le processus de décision?

(3) Comment prendre en compte, dans le modèle de préférences global, les dépendances qui ne peuvent être négligées?

La façon de répondre à ces questions est bien évidemment contingente au contexte décisionnel dans lequel se situe l'aide à la décision. Il me paraît néanmoins possible, en attirant l'attention sur un certain nombre de points, d'apporter des éléments de réponse utiles au modélisateur. Pour cela, je commencerai (en relation avec la première des trois questions ci-dessus) par interroger le concept de dépendances 
entre critères, ce qui m'amènera à faire quelques rappels. Dans la section suivante, je m'efforcerai d'apporter des éléments de réponse à la seconde des trois questions. J'examinerai enfin de quelle façon les dépendances entre critères qu'il est nécessaire de prendre en compte peuvent l'être à partir des principaux modèles d'agrégation actuellement utilisés. Je m'attarderai tout particulièrement sur le cas de l'intégrale de Choquet (cf. Sect. 4.2).

\section{SignificAtion ET Formes DE DÉPENDANCES}

Pour comprendre en quoi la présence de possibles « dépendances entre critères » peut être source de difficultés en aide à la décision $(\mathrm{AD})$, il m'est apparu nécessaire de commencer par préciser la signification de ce concept de dépendances lorsque l'on se préoccupe de construire un modèle de préférences global. Je reviendrai ensuite sur deux formes de dépendances qui sont envisagées de façon classique dans ce type de préoccupations.

\subsection{QUe SIGNIFIE « LES CRITÈRES DE $F$ NE SONT PAS INDÉPENDANTS $» ?$}

Cette affirmation est généralement présentée comme une source de difficultés, qu'elle soit prononcée par un théoricien ou par un praticien. Elle repose sur une reconnaissance de l'existence de liens entre ces critères, liens qui peuvent de nature très variée. Dans tous les cas, il s'agit, je crois, d'une mise en garde vis-à-vis de certains modes de raisonnement (notamment toutes choses égales par ailleurs) qui ignorent l'existence de ces liens. De façon plus précise, dans ce qui suit, je m'appuierai sur la définition suivante :

Définition 1 : La famille $F$ étant supposée construite et cohérente ( $c f$. Roy and Bouyssou [26], Chap. 2), dire qu'il existe des dépendances entre certains de ces critères signifie que l'on peut :

(1) mettre en évidence au moins deux sous-familles de $F$ (éventuellement réduites à un seul critère) entre lesquelles il existe des liens (de nature quelconque) tels que :

(2) ne pas tenir compte de ces liens pour asseoir la validité, la crédibilité ou l'intensité d'une relation de préférences globale peut conduire à négliger, involontairement et à tort, certains des aspects de la réalité qui méritent d'être pris en compte pour l'aide à la décision.

Avant d'illustrer cette définition, je précise que les préférences globales dont il est question ici peuvent indifféremment se rapporter à :

- un système de préférences supposé exister : démarche descriptive;

- un système de préférences accepté comme base de travail pour l'AD : démarche constructive.

Considérons un critère quelconque $g_{i}$ de $F$ (afin de simplifier les notations, on écrira aussi $i \in F$ ). Ce critère est, par définition ( $c f$. Roy and Bouyssou [26], Chap. 1), un instrument (ou fonction) qui permet d'associer à toute action potentielle $a$ une performance $g_{i}(a): g_{i}(a)$ peut être un nombre et, plus généralement, un élément 
(appelé ici échelon) d'un ensemble $E_{i}$ complètement ordonné, appelé échelle. Par convention, on dira que l'action $a$ est d'autant « meilleure » selon le critère $g_{i}$ que sa performance $g_{i}(a)$ est «plus élevée ». Par construction, chaque critère $g_{i}$ doit, vis-à-vis de tous les autres critères de $F$ jouir d'une propriété appelée séparabilité faible (cf. Grabisch [10]; Roy and Bouyssou [26], Sect. 2.4).

Définition 2 (propriété de séparabilité faible) : Pour tout $i \in F$ et toute paire d'actions $(a, b)$ vérifiant $g_{k}(a)=g_{k}(b), \forall k \neq i$, il ne peut y avoir qu'indifférence entre $a$ et $b$ ou préférence en faveur de $a$ vis-à-vis de $b$ dès l'instant où $g_{i}(a) \geqslant g_{i}(b)$ (autrement dit, $a \mathrm{~S} b: \ll a$ surclasse $b »$ ).

Cette propriété implique une forme d'indépendance qui est consubstantielle à la définition même du concept de critère : l'instrument qu'est un critère quel qu'il soit permet à lui seul de valider une relation de préférences globales entre deux actions quelconques dès l'instant où ces deux actions ont des performances identiques sur tous les autres critères et cela quoi qu'elles valent. Cette propriété autorise un mode de raisonnement «toutes choses égales par ailleurs ». Celui-ci est toutefois limité : la crédibilité ou l'intensité de la préférence $a$ vis-à-vis de $b$ peut dépendre des performances communes $g_{k}(a)=g_{k}(b), \forall k \neq i$, autrement dit la séparabilité faible n'implique nullement qu'il y ait indépendance (au sens de la définition 1) entre un critère $g_{i}$ et toute sous-famille de $F \backslash\{i\}$. L'exemple suivant met en évidence une telle forme de dépendance.

Exemple 1. Une valeur $v(a)$ doit être attribuée à toute action $a \in A$ à partir de deux critères, $g_{1}$ et $g_{2}$, chacun d'eux se rapportant à une qualité particulière que $a$ peut posséder à un niveau plus ou moins élevé. Les performances $g_{i}(a)$ sont évaluées sur une échelle commune allant de 0 à 100 (\%). On considère que la valeur de l'action est très fortement conditionnée par celle des deux qualités qui est la mieux satisfaite et, de façon très secondaire, par la façon dont l'autre l'est. Avec le décideur, il a été admis que les critères $g_{1}$ et $g_{2}$ étaient définis de façon à ce que toute égalité du type $g_{1}(a)=g_{2}(b)$ soit censée traduire une égale intensité de satisfaction de la propriété 1 par $a$ et de la propriété 2 par $b$. Dans ces conditions, il a été convenu de poser :

$$
v(a)=0.9 \max \left(g_{1}(a), g_{2}(a)\right)+0.1 \min \left(g_{1}(a), g_{2}(a)\right) .
$$

Le décideur a ensuite considéré que, pour $v(a) \geqslant v(b)$, la différence $v(a)-v(b)$ traduisait une intensité de préférence de $a$ vis-à-vis de $b$.

Considérons $a$ et $b$ telles que :

$$
g_{1}(a) \geqslant g_{1}(b) \quad \text { et } \quad g_{2}(a)=g_{2}(b)=x .
$$

Il est clair que l'intensité de préférence dépend ici de la façon dont la valeur commune $x$ se situe par rapport à l'intervalle $\left(g_{1}(b), g_{1}(a)\right)$. Selon cette valeur de $x$, l'intensité peut varier de $0.9\left(g_{1}(a)-g_{1}(b)\right)$ à $0.1\left(g_{1}(a)-g_{1}(b)\right)$. 


\section{2. À PRopos DE DEux Formes Classiques DE DÉPENDANCES}

\subsubsection{Dépendances au sens structurel}

Pour le non initié, cette forme de dépendances est la seule qu'il envisage. Souvent, il l'évoque spontanément. Elle provient de la présence de facteurs susceptibles d'influencer conjointement plusieurs critères. Cette forme de dépendances doit normalement pouvoir être mise en évidence en examinant la façon dont les critères ont été construits : présence de données communes ou encore significativement corrélées. Il importe de ne pas perdre de vue que les éventuelles corrélations que peuvent mettre en évidence des outils statistiques appliqués au tableau des performances défini sur un ensemble $A$ fixé peuvent être très contingentes à cet ensemble et ne pas être pertinentes pour l'aide à la décision.

Soit $g_{h}$ et $g_{j}$ deux critères concernés par cette forme de dépendances. Ne pas tenir compte, dans la modélisation des préférences globales, des liens qui peuvent en découler, c'est ignorer le fait que le ou les facteurs qui influencent conjointement les performances $g_{h}(a)$ et $g_{j}(a)$ sont pris en compte d'une part par $g_{h}$ et, d'autre part, par $g_{j}$. La question qu'il convient de se poser est alors la suivante :

Ce fait est-il un aspect de la réalité qui mérite d'être pris en compte pour l'aide à la décision? Peut-il, si on le néglige, provoquer des «doubles comptes » malencontreux?

La réponse peut être non. Ce sera notamment le cas lorsque les critères $g_{h}$ et $g_{j}$ traduisent des points de vue fondamentalement différents ou encore lorsqu'ils affectent des parties prenantes distinctes.

Exemple 2. Le coût d'investissement et le gain de temps des usagers sont deux critères importants pour comparer des variantes de tracés de voie rapide ou de lignes TGV devant relier la même paire d'agglomérations. La longueur du tracé est un facteur qui influence chacun de ces critères. La modélisation des préférences globales peut fort bien l'ignorer ; le premier critère concerne l'investisseur, le second les usagers. Le facteur commun ne crée ni redondance (double compte), ni synergie positive (complémentarité).

La réponse peut aussi être oui (voir des exemples dans Bisdorff [4]; Roy [23], Chap. 10; Roy and Bouyssou [26], Chap. 2). Les développements qui suivent (essentiellement en Sect. 4) mettront en évidence quelques solutions possibles (non exemptes de difficultés) pour prendre explicitement en compte cette forme de dépendances dans la modélisation des préférences globales. Il ne faut pas perdre de vue qu'une façon souvent plus simple de surmonter les difficultés qu'elles créent consiste à modifier la définition de la famille de critères $F$. Dans certains cas, on pourra tout simplement substituer, aux critères $g_{h}$ et $g_{j}$, un unique critère. Dans d'autres cas, il faudra modifier la définition de l'un (voire des deux critères) concerné et compléter cette nouvelle paire de critères par un (éventuellement plusieurs) critère supplémentaire. 


\subsubsection{Dépendances au sens des préférences}

Bien que classique, il est une autre forme de dépendances que le non initié a souvent beaucoup de mal à comprendre. Sa présence tient au fait que certains modes de raisonnement (souvent implicites) toutes choses égales par ailleurs qui font intervenir au moins trois critères doivent être proscrits dans la façon de concevoir le modèle de préférences globales.

Par définition, on dit qu'une sous-famille $J$ d'au moins deux critères $(J \subset F)$ est dépendante au sens des préférences dans $F$ si la façon de comparer deux actions $a$ et $b$ vérifiant :

$$
g_{k}(a)=g_{k}(b), \forall k \in F \backslash J
$$

est susceptible d'être influencée par les performances de $a$ et $b$ sur les critères n'appartenant pas à $J$ (bien que ces performances soient les mêmes).

Exemple 3. Le responsable d'un service technique a retenu les trois critères suivants pour sélectionner, parmi un ensemble de candidats, celui qu'il va embaucher :

$g_{1}:$ niveau de formation initiale;

$g_{2}$ : avis sur l'aptitude à une bonne intégration dans son équipe;

$g_{3}$ : nombre d'années d'expérience professionnelle.

Soit $a$ et $b$ deux candidats ex aquo sur le critère $g_{3}$ et tels que :

$$
\begin{aligned}
& g_{1}(a)=\text { excellent } ; g_{2}(a)=\text { quelques réserves, } \\
& g_{1}(b)=\text { bon } ; g_{2}(b)=\text { aucune réserve. }
\end{aligned}
$$

Avec $g_{3}(a)=g_{3}(b)=1$, le responsable peut préférer $a$ à $b$ alors que, avec $g_{3}(a)=$ $g_{3}(b)=5$, il peut préférer $b$ à $a$. Dans ces conditions, la famille $\left\{g_{1}, g_{2}\right\}$ n'est pas indépendante des préférences dans $F$.

Le lecteur trouvera d'autres exemples dans Grabisch [9], Grabisch and Labreuche [11], Grabisch and Perny [14], Keeney and Raiffa [18], Roy [23], Roy and Bouyssou [26]. Dans la suite de cet article, je montrerai que cette forme de dépendances est difficile à diagnostiquer, à rendre intelligible et plus encore à prendre clairement en compte dans un modèle de préférences global.

\section{Mise EN ÉvidenCE ET INTELLIGIBILITÉ DES DÉPENDANCES}

En pratique, il s'avère souvent difficile de prendre position sur le fait que $F$ (une fois construite) peut ou ne peut pas être traitée comme étant dépourvue de toutes formes de dépendances entre ces critères au sens de la Définition 1. Comme on le verra en Section 4, les modèles d'agrégation les plus couramment utilisés supposent cette indépendance. Pour ces deux raisons, en aide à la décision, on s'efforce généralement de construire $F$ de telle sorte que les dépendances, s'il en existe, n'aient pas à être prises en compte ( $c f$. Ex. 2). Dans certains cas, il est toutefois nécessaire de s'assurer qu'il en est bien ainsi. Dans ce but, l'équipe d'étude conçoit généralement un mode de questionnement apte aussi bien à tester cette 
indépendance qu'à mettre en évidence les éventuelles dépendances qui pourraient exister.

Dans la présente section, je commencerai par examiner les conditions qui doivent être satisfaites pour que les réponses obtenues soient probantes. Je montrerai ensuite qu'il peut être malaisé de cerner certaines formes de dépendances. Je mettrai finalement en évidence d'autres formes qui sont à la fois aisées à diagnostiquer et à faire comprendre.

\subsection{Probance Des RÉPonses}

Je voudrais tout d'abord faire remarquer que, pour mettre en évidence l'existence de possibles dépendances, le mode de questionnement envisagé ci-après n'est pas la seule approche concevable. Une analyse méthodique des données prises en compte par chaque critère jointe à la façon dont les critères ont été construits peut, dans certains cas, fournir les réponses cherchées. Il ne faut cependant pas perdre de vue que les dépendances susceptibles d'être mises en évidence par l'emploi d'outils statistiques (notamment l'analyse de données) n'est généralement pas immédiatement interprétable en termes de préférences.

Envisager un mode de questionnement implique en premier lieu d'identifier la ou les personnes à interroger. Dans ce qui suit, je parlerai de l'interrogé, même s'il y a plusieurs personnes. Cet interrogé devrait normalement être «le décideur » (autrement dit celle ou celui pour qui ou au nom de qui l'AD s'exerce) ou, à défaut, quelqu'un qui le représente. Cela peut ne pas être possible. C'est alors l'équipe d'étude elle-même qui joue ce rôle. Même si, dans ce dernier cas, elle peut être involontairement amenée à justifier ce qu'elle a envie de trouver, c'est, à défaut de mieux, une démarche non dénuée de pertinence.

En second lieu, l'équipe d'étude doit concevoir un protocole d'interrogation approprié au but visé. Les réponses obtenues seront d'autant plus probantes que les hypothèses suivantes seront mieux satisfaites.

Hypothèse 1.a (cas d'une démarche descriptive) : Les réponses fournies par l'interrogé sont dictées par un système de préférences global préexistant, lequel demeure invariant tout au long du questionnement.

Hypothèse 1.b (cas d'une démarche constructive) : Les réponses fournies par l'interrogé sont dictées par des propriétés que le système de préférences global doit satisfaire pour qu'il puisse le juger approprié au processus d'AD.

Hypothèse 2 : L'interrogé est apte à replacer, dans le contexte concret concerné, les questions (même si elles sont nombreuses et complexes) qu'il a été jugé nécessaire de lui poser afin que l'ensemble des réponses obtenues soit suffisamment riche pour permettre qu'un traitement approprié puisse mettre en évidence, de façon intelligible et significative, la présence éventuelle de dépendances.

J'examine rapidement ci-après les principales raisons qui peuvent contribuer à rendre ces hypothèses plus ou moins bien satisfaites. 
Plaçons-nous en premier lieu dans le cas d'une démarche descriptive. Si l'interrogé appartient à l'équipe d'étude, le système de préférences préexistant qui dicte ses réponses peut être significativement différent de celui du « décideur . Quoi qu'il en soit, pour pouvoir replacer correctement, dans le contexte concret concerné, les questions qui lui sont posées, il lui faut non seulement connaître ce contexte dans tout ce qu'il a d'objectif mais aussi être en accord avec la façon subjective dont le décideur les appréhende. Si l'interrogé n'appartient pas à l'équipe d'étude, les réponses peuvent, pour une part, être fabriquées par le questionnement et n'être qu'en apparence dictées par un système de préférences préexistant, cela notamment pour les raisons suivantes :

(i) Les préférences qui préexistent au questionnement peuvent être incomplètes, mal définies, présenter d'éventuelles incohérences, ce qui peut rendre certaines réponses non probantes.

(ii) La nouveauté des questions posées peut amener l'interrogé à réviser momentanément et localement certaines de ses préférences.

(iii) Certaines réponses peuvent être arbitraires, soit parce que la complexité de la question la rend difficile à comprendre ou à replacer dans le contexte concret, soit parce que le mode de questionnement enferme l'interrogé dans un dilemme (oui ou non, $a$ mieux que $b$ ou $b$ mieux que $a, \ldots$ ).

(iv) La complexité et le nombre des questions peuvent assez rapidement occasionner une fatigue qui rend les réponses empreintes d'une part d'aléatoire.

Voir aussi Roy [24].

Plaçons-nous en second lieu dans le cas d'une démarche constructive. Le questionnement n'est plus destiné à asseoir un modèle qui reflète aussi fidèlement que possible une réalité préexistante. Il a pour objet la mise à jour d'hypothèses sur la base desquelles le modèle construit pourra jouer le rôle qui lui est dévolu dans le processus d'AD (outils pour approfondir, interpréter, expérimenter, débattre,... ). Les questions posées doivent ici être aptes à mettre en évidence les liens qui méritent d'être pris en compte pour l'AD. Dans ce but, il est souvent préférable que l'interrogé ne soit pas réduit à un seul individu. Les raisons (i) à (iv) évoquées ci-dessus peuvent ici encore affecter la probance des réponses (que l'interrogé soit dans ou hors de l'équipe d'étude) si celles-ci ne sont pas soumises à un examen critique rigoureux.

\subsection{DES DÉPENDANCES DIFFICILES À CERNER}

Le mode de questionnement auquel je viens de me référer est en fait souvent utilisé pour appréhender les dépendances par une voie indirecte. Il vise à attribuer des valeurs aux paramètres d'un modèle a priori apte à prendre en compte certaines formes de dépendances (éventuellement non clairement explicitées ; cf. [11,18,26]). Les dépendances ainsi cernées ne le sont pas pour autant en termes directement intelligibles, surtout si les paramètres qui servent à les appréhender n'ont pas une signification concrète bien claire. Je reviendrai sur ce sujet en Section 4.

Pour les raisons exposées ci-dessus, les hypothèses 1 et 2 sont généralement assez mal vérifiées en pratique. Quoi qu'il en soit, il importe de ne pas perdre de 
vue que les parties prenantes qu'il s'agit d'aider avec le modèle en question veulent souvent comprendre la signification de ces dépendances. Dans la mesure où cellesci viennent fortement compliquer le modèle, il convient aussi (surtout lorsqu'elles sont établies de façon peu probante) de s'assurer que leur prise en compte effective se traduit autrement que par des raffinements illusoires.

L'insuffisance d'intelligibilité et le risque de raffinements illusoires sont à redouter d'autant plus que les deux familles de critères entre lesquelles des liens de dépendances doivent être appréhendés font intervenir un nombre de critères plus élevé. À plusieurs reprises, j'ai eu l'occasion de constater que, dès lors qu'au moins trois critères doivent intervenir conjointement (comme dans l'Ex. 3), la mise au point d'un questionnement direct pouvait être malaisée. En l'absence d'exemples illustrant clairement un cas où cette forme de dépendances était manifeste, l'interrogé ne la comprenant que très mal donnait, dans le contexte étudié, des réponses telles que cette forme de dépendances n'avait pas lieu d'être prise en compte. En revanche, suite à quelques exemples choisis pour mettre en évidence le cas de liens similaires à ceux pouvant exister dans le contexte étudié, les réponses pouvaient devenir tout à fait différentes.

\subsection{Des DÉPEndances intelligibles ET FACILES À CERNER}

Heureusement, beaucoup de cas de dépendances qu'il semble utile de devoir prendre effectivement en compte pour l'AD ne portent que sur des couples de critères.

Des échanges oraux avec José Figueira et Salvatore Greco m'ont amenés à m'intéresser à trois types de situations faisant intervenir de telles dépendances susceptibles d'être clairement décrites en ne faisant intervenir que deux critères. Dans un contexte donné, la présence de couples sur lesquels elles peuvent porter doit, me semble-t-il (bien je ne l'ai pas encore expérimenté), être faciles à diagnostiquer. En outre, il devrait être relativement aisé de juger s'il convient ou non de prendre explicitement en compte ces dépendances dans le modèle de préférences global. En accord avec José Figueira et Salvatore Greco, je propose de caractériser ces situations comme suit.

\section{Situation d'auto-renforcement entre $g_{h}$ et $g_{j}$}

On considère que, dès l'instant où deux actions $a$ et $b$ sont telles que « $a$ est aussi bonne que $b »$ sur chacun des critères $g_{h}$ et $g_{j}$, cela doit entraîner un renforcement de l'impact qu'ont ces critères pour asseoir la relation de préférences globale : plus précisément, la façon dont ces critères contribuent à la validité, à la crédibilité ou à l'intensité de cette relation doit être plus significative que celle obtenue par simple addition des deux impacts propres à chacun de ces critères lorsque (toutes choses égales par ailleurs) il est seul à valider l'assertion « $a$ est au moins aussi bonne que $b »$. 


\section{Situation d'auto-affaiblissement entre $g_{h}$ et $g_{j}$}

On considère que, dès l'instant où deux actions $a$ et $b$ sont telles que « $a$ est aussi bonne que $b »$ sur chacun des critères $g_{h}$ et $g_{j}$, cela doit entraîner un affaiblissement de l'impact qu'ont ces critères pour asseoir la relation de préférences globale : plus précisément, la façon dont ces critères contribuent à la validité, à la crédibilité ou à l'intensité de cette relation doit être moins significative que celle obtenue par simple addition des deux impacts propres à chacun de ces critères lorsque (toutes choses égales par ailleurs) il est seul à valider l'assertion « $a$ est au moins aussi bonne que $b »$.

\section{Situation d'antagonisme de $g_{j}$ vis-à-vis de $g_{h}$}

On considère que, dès l'instant où deux actions $a$ et $b$ sont telles que $« a$ est au moins aussi bonne que $b »$ sur le critère $g_{h}$ alors que $\ll b$ est significativement préférée à $a »$ sur le critère $g_{j}$, cela doit entraîner une réduction de l'impact qu'a le critère $g_{h}$ pour asseoir la relation de préférences globale : plus précisément, la façon dont ce critère contribue à la validité, à la crédibilité ou à l'intensité de cette relation doit être moins significative que ce qu'elle est lorsque (toutes choses égales par ailleurs) le critère $g_{j}$ ne valide pas l'assertion $\ll b$ est significativement préférée à $a »$.

Ces définitions appellent les remarques suivantes :

(a) Les situations d'auto-renforcement et d'auto-affaiblissement concernent des formes particulières de ce que l'on appelle les effets de synergies (respectivement positive et négative). Soulignons que ces formes de synergies positive et négative ne doivent pas être confondues avec celles que modélise l'interaction entre deux critères dans l'intégrale de Choquet ( $c f$. par exemple [14]). Je reviendrai sur cette différence au 4.2. La situation d'antagonisme concerne ce que l'on peut appeler un effet de sape.

(b) Chacune des trois définitions ci-dessus n'a de sens que si $F$ n'est pas réduite aux deux seuls critères $g_{h}$ et $g_{j}$.

(c) Ces définitions ne sous-entendent aucune hypothèse quant à la nature des échelles concernées : $E_{h}$ et $E_{j}$ peuvent être des échelles purement ordinales non nécessairement identiques.

(d) Les situations d'auto-renforcement et d'auto-affaiblissement portent sur la paire $\left\{g_{h}, g_{j}\right\}$ alors que la situation d'antagonisme porte sur le couple $\left(g_{h}, g_{j}\right)$ : dans cette dernière situation, les deux critères ne jouent pas le même rôle.

(e) Pour une paire $\left\{g_{h}, g_{j}\right\}$, il ne peut y avoir à la fois auto-renforcement et auto-affaiblissement.

(f) La présence d'un antagonisme pour le couple $\left(g_{h}, g_{j}\right)$ n'implique ni n'exclut la présence d'un antagonisme pour le couple $\left(g_{j}, g_{h}\right)$.

Exemple 4. Revenons à l'Exemple 1. Supposons maintenant que, pour définir $v(a)$, il faille également prendre en compte la performance $g_{3}(a)$ sur un troisième 
critère (l'échelle associée étant la même que pour $g_{1}$ et $g_{2}$ ). Examinons les deux possibilités suivantes.

(a) $g_{3}$ a, sur $v(a)$, un impact additif qui ne dépend ni de $g_{1}$, ni de $g_{2}$. On est alors fondé à poser :

$v(a)=0.9 \max \left(g_{1}(a), g_{2}(a)\right)+0.1 \min \left(g_{1}(a), g_{2}(a)\right)+p \cdot g_{3}(a), p>0$.

On remarquera que, dans ce modèle d'agrégation, la forme de dépendances qui existe entre $g_{1}$ et $g_{2}$ ne correspond à aucune des trois situations définies ci-dessus.

(b) $g_{3}$ a, sur $v(a)$, un impact additif qui n'est pas indépendant de $g_{2}$ en ce sens qu'il y a auto-renforcement entre $g_{2}$ et $g_{3}$. On pourrait par exemple envisager de poser :

$v(a)=0.9 \max \left(g_{1}(a), g_{2}(a)\right)+0.1 \min \left(g_{1}(a), g_{2}(a)\right)+p \cdot g_{2}(a) \cdot g_{3}(a), p>0$.

Ce modèle de préférences traduit certes une forme de synergie positive entre $g_{2}$ et $g_{3}$ mais elle ne correspond pas à celle d'auto-renforcement définie ci-dessus. Considérons en effet deux actions $a$ et $b$ ayant mêmes performances sur $g_{1}$. Plaçonsnous par exemple dans le cas $g_{1}(a)=g_{1}(b) \geqslant g_{2}(a)>g_{2}(b)$. Il vient :

$$
v(a)-v(b)=0.1\left[g_{2}(a)-g_{2}(b)\right]+p\left[g_{2}(a) \cdot g_{3}(a)-g_{2}(b) \cdot g_{3}(b)\right] .
$$

Dans les conditions qui ont conduit à l'égalité (3.3), il apparaît clairement que la forme de synergie modélisée dans la formule (3.2) influence la façon dont $a$ se compare à $b$ même lorsque $g_{3}(b)<g_{3}(a)$, ce qui n'est pas conforme à la situation d'auto-renforcement.

Le lecteur trouvera, dans Figueira et al. [6], deux exemples concrets illustrant les trois situations définies ci-dessus.

Pour terminer, je crois utile d'attirer l'attention sur le fait qu'aucune de ces trois situations ne peut être prise en compte lorsque les performances sont agrégées dans un critère de synthèse (fonctions de valeurs, fonctions d'utilités, scores,...). En effet, la valeur $v(a)$ que ce critère attribue à une action $a$ quelconque ne prend en compte que les performances de la seule action $a$ sur les différents critères. Tout modèle de préférence fondé sur la comparaison des valeurs $v(a)$ et $v(b)$ est donc inapte à prendre en compte un lien de dépendance qui est conditionné par le fait que $a$ et $b$ se comparent ou non de la même façon sur deux critères entre lesquels ce lien doit exister. Or, pour modéliser chacune des trois situations ci-dessus, il doit en être ainsi. Ceci n'est possible que si l'on opère d'abord une comparaison entre $a$ et $b$ critère par critère pour effectuer ensuite l'agrégation; ceci a entre autres le mérite de pouvoir prendre aisément en compte des seuils de discrimination (indifférence et préférence) et des seuils de veto. 


\section{DÉPEndances et PRocÉdures D'AGRÉGation}

\section{1. À PROPOS DES MODES D'AGRÉGATION USUELS}

Considérons tout d'abord les modèles analytiques d'agrégation les plus couramment utilisés en aide à la décision, notamment AHP, ELECTRE, PROMETHEE, MACBETH, MAUT (version élémentaire), TOPSIS ( $c f .[5])$. Ces modèles ont été conçus pour prendre en compte une famille $F$ de critères lorsqu'il est légitime de supposer qu'il n'existe aucune forme de dépendance entre ces critères (au sens de la Déf. 1). Il convient toutefois de préciser que MAUT, sous sa forme la plus générale ( $c f$. [18]), est une théorie qui peut s'affranchir de cette hypothèse. Elle est même accompagnée de tests ayant pour objet de la valider ou de l'infirmer. Pour les raisons exposées au 3.1, ces tests risquent de n'être que rarement probants. Quoi qu'il en soit, vouloir utiliser en pratique MAUT pour prendre explicitement en compte quelques formes de dépendances que ce soit (notamment des dépendances au sens des préférences) risque fort de conduire à un modèle compliqué, difficile à décoder en termes concrets. En outre, pour les raisons exposées à la fin de la section précédente, il est inapte à prendre en compte des situations de dépendances du type de celles définies à la section 3.3.

Ces trois situations ont pu en revanche être aisément prises en compte dans les méthodes ELECTRE grâce à une extension de la définition de l'indice de concordance $(c f .[6])$. Cette nouvelle définition reste compatible avec la présence de pseudo-critères et d'échelles purement ordinales.

Alors que les modèles analytiques susceptibles d'être utilisés pour définir un critère de synthèse attribuant une valeur à chaque action sont inaptes à prendre en compte certaines formes de dépendances ( $c f$. fin de la section précédente), les modèles qui définissent l'agrégation à partir d'un système de règles ( $c f$. $[2,3,15,17,20,23,27,28])$ sont a priori aptes à prendre explicitement en compte n'importe quelle forme de dépendance. Ici encore, la distinction entre démarche descriptive et démarche constructive est importante d'un point de vue opérationnel. Dans le premier cas, expliciter les règles qui rendent compte de situations de préférence décrites a priori peut conduire à un système de règles extrêmement complexes, difficilement intelligibles, donc la cohérence peut ne pas être automatiquement garantie par la façon dont elles sont mises en évidence. Dans le second cas, il s'agit essentiellement de s'assurer de cette cohérence entre les règles qui sont a priori postulées (celles-ci étant, par définition, intelligibles).

Je vais consacrer la fin de la présente section à l'intégrale de Choquet comme modèle analytique permettant de construire une fonction d'utilité apte à prendre en compte certaines formes de dépendances (souvent appelées interactions entre critères). D'autres modèles d'utilité le permettent également (voir notamment $[7,18,22])$. Si l'intégrale de Choquet retient ici plus particulièrement mon attention, c'est en raison des nombreuses publications qui, depuis quelques temps, en font état pour l'aide à la décision. 


\subsection{Portées et limites de l'intégrale de Choquet pour l’aide À LA DÉCISION}

Je me limiterai ici à un seul usage de l'intégrale de Choquet (cf. $[8,10,14,19,21])$ : agrégation de critères pour construire une fonction d'utilité destinée à l'aide à la décision (je rappelle que l'intégrale de Choquet n'a pas été conçue dans ce but et qu'elle peut être utilisée autrement en aide à la décision), par exemple dans une méthode de type ELECTRE pour définir un indice de concordance global ( $c f$. [14]).

Je commencerai par attirer l'attention sur les prérequis indispensables pour cet usage de l'intégrale de Choquet.

En premier lieu, à chacun des critères $g_{i}$ de la famille $F$, on doit faire correspondre (pour lui être substituée dans les calculs) une fonction d'utilité $u_{i}$ qui place les $u_{i}(a)$ sur une même échelle de ratio $E$.

Ceci implique :

(1) Toute égalité du type $u_{i}(a)-u_{j}(a)=0$ doit pouvoir être interprétée comme suit : l'action a procure exactement la même intensité de satisfaction avec les critères $g_{i}$ et $g_{j}$ : propriété de commensurabilité des performances (cf. [14], Sect. 3.4.1).

(2) Toute inégalité du type $u_{i}(a)-u_{i}(b)>0$ doit pouvoir être interprétée comme : un avantage significatif indiscutable en faveur de a vis-à-vis $d e b$. Ceci exclut toute possibilité de prise en compte, dans la fonction d'utilité $u_{i}$, d'éventuels seuils d'indifférence et de préférence qui affecteraient les performances $g_{i}(a)$ en raison d'incertitudes, d'imprécisions ou de mauvaises déterminations. En pratique, cette impossibilité de prendre en compte le caractère non significatif de petits écarts peut conduire à ce que leur cumul, sur une sous-famille de critères, vienne compenser un écart réellement significatif de sens opposé sur un autre critère.

Dans ce qui suit, je suppose que les fonctions d'utilité ont pu être construites. Ceci peut bien évidemment se heurter à de réelles difficultés lorsque les performances $g_{i}(a)$ se situent sur des échelles $E_{i}$ qui se rapportent à des réalités concrètes très différentes (voir les exemples 2 et 3 ci-dessus, les exemples 1 à 12 dans Roy [23] ou encore ceux étudiés aux chapitres 8-10 dans Roy and Bouyssou [26]). Pour construire ces fonctions, Grabisch [10] propose d'utiliser MACBETH. Dans cette méthode, le protocole d'interrogation a été conçu dans l'hypothèse où il n'existait aucune forme de dépendances entre critères. Il doit permettre de construire une fonction d'agrégation de type somme pondérée qui replace les performances sur des échelles d'intervalle $E_{i}$. Pour pouvoir utiliser ce protocole en présence d'éventuelles dépendances afin de mettre en évidence les fonctions d'utilité $u_{i}$ définies sur une échelle de ratio commune $E$, je crois nécessaire de chercher à expliciter (en relation avec ce qui est dit section 3.1) les éventuelles hypothèses supplémentaires que cela implique pour garantir la validité de cette façon de faire.

Afin de simplifier le langage, je conserve ci-après le terme performance pour désigner aussi bien $u_{i}(a)$ que $g_{i}(a)$. En revanche, je réserve le terme critère à la 
fonction $g_{i}$ telle qu'elle a été initialement définie tout en continuant à parler du critère $i \in F$.

En second lieu, à chacun des $2^{n}$ sous-ensembles $J \subset F$, on doit attribuer une valeur $\mu(J)$, appelée capacité, en respectant les conditions suivantes :

$$
\mu(\varnothing)=0, J \subset H \rightarrow \mu(J) \leqslant \mu(H), \mu(F)=1 .
$$

Je crois utile d'attirer l'attention sur le fait que j'utilise ici le terme capacités pour désigner les valeurs attribuées à $\mu(J), \forall J \subseteq F$; $\mu$ désigne la fonction qui est ainsi construite à partir de ces valeurs. Cette fonction, qui est également appelée capacité, n'est pas ici une donnée qui définirait des valeurs $\mu(J)$ : ces valeurs doivent être construites. Je reviendrai plus loin sur les questions qui se posent à ce sujet. Une fois ces capacités construites, l'intégrale de Choquet conduit à attribuer, à chaque action $a$, une utilité $u(a)$ définie comme suit :

$$
u(a)=\sum_{r=1}^{n}\left[\mu\left(J_{r}\right)-\mu\left(J_{r-1}\right)\right] u_{(r)}(a)
$$

avec $J_{r}$ : ensemble des critères qui occupent un rang au plus égal à $r$ dans un rangement des critères qui place les performances de $a$ par valeurs non croissantes, $u_{(r)}(a)$ désignant la performance de $a$ selon le critère qui occupe le rang $r$ dans ce rangement.

Dans ce modèle, la capacité $\mu(J)$ s'interprète comme l'utilité d'une action $a^{J}$ qui serait telle que :

$$
u_{i}\left(a^{J}\right)=e^{*}, \forall i \in J \text { et } u_{i}\left(a^{J}\right)=e_{*}, \forall i \notin J
$$

$e^{*}$ et $e_{*}$ désignant respectivement les échelons supérieurs et inférieurs de $E$ (pour une axiomatisation de ce modèle, voir Grabisch [12]; Labreuche and Grabisch [19]).

Dans ce modèle, ce sont les valeurs attribuées aux $2^{n}-2$ capacités (relatives au sous-ensemble propre de $F$ ) qui prennent en compte les éventuelles dépendances (on parle aussi d'interactions; $c f$. Grabisch and Perny [14]). L'absence de dépendance est caractérisée par des capacités additives, c'est-à-dire telles que, $\forall J \subset$ $F: \mu(J)=\sum_{i \in J} \mu\left(g_{i}\right)$. Il s'ensuit que, utiliser l'intégrale de Choquet pour prendre en compte d'éventuelles dépendances n'est possible que si l'on sait définir les $2^{n}-2$ capacités de façon appropriée.

Après la construction des fonctions d'utilité $u_{i}$, celle des capacités $\mu(J)$ constitue l'aspect crucial de l'usage de l'intégrale de Choquet pour prendre en compte d'éventuelles dépendances. Grabisch [8] présente trois approches (pour plus de détails et pour d'autres approches éventuelles, voir Angilella et al. [1]; Grabisch and Labreuche [11]; Marichal and Roubens [21]). Je me borne à en donner une présentation très succincte ci-après.

La première consiste à tirer parti des relations (4.1) et (4.3), de l'existence possible de certaines symétries ou encore d'éventuels effets de veto ainsi que de 
la définition de certains indices tels la valeur de Shapley (qui peut s'interpréter comme un degré d'importance de chaque critère compte tenu des interactions; $c f$. par exemple [14]) ou encore ceux d'interaction, de pouvoir améliorant moyen, de conjonction, de disjonction. En fait, cette première approche permet essentiellement de diminuer le nombre des capacités qu'il est nécessaire de déterminer a priori (les autres pouvant en être déduites).

La seconde approche repose sur la possibilité de faire indiquer a priori, par le décideur ou quelqu'un qui le représente, la valeur qu'il souhaiterait que le modèle attribue à des actions de référence (ou, à défaut, un préordre complet sur cet ensemble d'actions). Si ces actions de référence sont suffisamment nombreuses et bien choisies, il est possible (par résolution d'un programme quadratique) de déterminer les capacités qui minimisent la somme des carrés des écarts entre valeurs indiquées et valeurs calculées par la formule (4.2) (d'autres critères d'ajustement sont également proposés).

La troisième approche consiste simplement à combiner les deux précédentes afin de simplifier les calculs que réclame la seconde. Demander à un interrogé d'attribuer des valeurs (sur une échelle de ratio) à des actions de référence qui ne peuvent être quelconques peut s'avérer très difficile. Pour y parvenir, on peut commencer par faire exprimer des comparaisons portant sur des paires d'actions de façon à tenter de bâtir un préordre complet sur l'ensemble des actions de référence.

Quelle que soit la façon de procéder, il me paraît indispensable de cherche à s'assurer :

- de la probance des données issues des réponses à un questionnement ( $c f$. Sect. 3.1 (i)-(iv));

- du caractère significatif des capacités trouvées, tout d'abord en testant leur « robustesse » face à des variations raisonnables des données, puis en vérifiant que, en s'écartant légèrement du minimum obtenu avec le critère d'ajustement retenu, on ne trouve pas des capacités significativement différentes car, si tel est le cas, ces nouvelles capacités constituent une solution aussi pertinente que celle qui garantit le minimum absolu.

Je vais maintenant mettre en évidence certaines des difficultés auxquelles on se heurte (une fois les fonctions d'utilité $u_{i}$ construites) pour prendre en compte, de façon probante et intelligible, des dépendances (ou interactions) entre critères. J'examinerai pour cela le cas d'une démarche descriptive puis celui d'une démarche constructive.

\section{(a) Cas d'une démarche descriptive}

Les procédures existantes rappelées ci-dessus concernent principalement ce cas. Dès que $n$ dépasse quelques unités, il peut être difficile de s'assurer de la probance des réponses fournies par l'interrogé ainsi que de la robustesse des valeurs que la procédure d'ajustement attribue aux capacités. Ces valeurs une fois fixées, je crains qu'il n'y ait pas de réponses simples aux deux questions suivantes :

- Comment rendre intelligibles, par les parties prenantes au processus d'AD, les dépendances effectivement prises en compte par ces capacités? 
- Comment s'assurer que l'utilité $u(a)$ définie par la formule (4.2) ne fabrique pas, pour certains couples $(a, b)$ d'actions, des résultats non conformes à l'opinion de l'interrogé, résultats pouvant concerner le signe de $u(a)-u(b)$ ou encore la façon dont cette différence dépend de certaines performances? La réponse à cette question est certes difficile dans beaucoup de modèles, tout spécialement lorsque $n$ est grand. Comme je vais le montrer ci-après, la formule (4.2) (de par sa nature) oblige, pour chercher une réponse à ce type de question, à envisager un très grand nombre de cas, même lorsque $n$ est petit (voir aussi les exemples présentés en annexe dans Roy [25], relatifs à un cas particulier d'intégrale de Choquet).

Exemple 5. Considérons le cas de trois critères $g_{1}, g_{2}, g_{3}(E=[0,20])$. Supposons que la résolution du programme quadratique mentionné ci-dessus ait conduit à retenir les valeurs suivantes :

$$
\mu\left(g_{1}\right)=0.4, \mu\left(g_{2}\right)=0.5, \mu\left(g_{1}, g_{2}\right)=0.95, \mu\left(g_{2}, g_{3}\right)=0.9
$$

Considérons les quatre actions suivantes :

$$
\begin{aligned}
& a_{1}=(16,13,7), a_{2}=(16,13,13), \\
& b_{1}=(7,19,7), b_{2}=(7,19,13) .
\end{aligned}
$$

Avec la formule (4.2), on obtient :

$$
\begin{aligned}
& u\left(a_{1}\right)=13.9, u\left(a_{2}\right)=14.2, \\
& u\left(b_{1}\right)=13, u\left(b_{2}\right)=15.4 .
\end{aligned}
$$

$a_{1}$ est donc préférée à $b_{1}$ alors que $b_{2}$ est préférée à $a_{2}$. Ces deux cas de dépendances au sens des préférences reflètent-ils les préférences de l'interrogé ou sont-ils un artéfact fabriqué par le modèle? N'y a-t-il pas beaucoup d'autres cas semblables qui risquent de passer inaperçus?

Plus généralement, considérons, comme dans l'exemple ci-dessus, deux actions $a$ et $b$ telles que, pour un certain critère $k, u_{k}(a)=u_{k}(b)=x$. Soit $r$ et $s$ les rangs respectifs qu'occupe cette performance $x$ dans les rangements des performances de $a$ et de $b$ par valeurs non croissantes. D'après la formule (4.2), on peut mettre les utilités respectives de $a$ et de $b$ sous la forme suivante :

$$
u(a)=A_{r}+\alpha_{r} \times x, u(b)=B_{s}+\beta_{s} \times x .
$$

Lorsque $x$ varie sans que les rangs $r$ et $s$ soient modifiés, la différence $u(a)-u(b)$ ne demeure inchangée que si $\alpha_{r}=\beta_{s}$. Si $\alpha_{r} \neq \beta_{s}, u(a)-u(b)$ dépend de $x$ et rien ne s'oppose à ce que cette différence change de signe lorsque $x$ décrit l'intervalle autorisé. Lorsque $x$ sort de cet intervalle, c'est-à-dire occupe un rang autre que $r$ et/ou $s$ (ce qui est le cas dans l'exemple ci-dessus), les termes $A_{r}$ et/ou $B_{s}$ ainsi que les coefficients $\alpha_{r}$ et/ou $\beta_{s}$ s'en trouvent affectés. Cette brève analyse montre à quel point il peut être difficile de mettre en évidence les cas de dépendances au 
sens des préférences que l'intégrale de Choquet modélise compte tenu des valeurs attribuées aux capacités.

Supposons maintenant que les valeurs attribuées aux capacités l'aient été à partir des six actions de référence suivantes :

\begin{tabular}{|l|l|l|l|l|}
\hline Actions & $u_{1}$ & $u_{2}$ & $u_{3}$ & $u\left(r_{j}\right)$ \\
\hline$r_{1}$ & 16 & 6 & 6 & 10 \\
\hline$r_{2}$ & 6 & 16 & 6 & 11 \\
\hline$r_{3}$ & 6 & 6 & 16 & 8 \\
\hline$r_{4}$ & 16 & 16 & 6 & 15.5 \\
\hline$r_{5}$ & 6 & 16 & 16 & 15 \\
\hline$r_{6}$ & 16 & 6 & 16 & 12 \\
\hline
\end{tabular}

L'intégrale de Choquet (formule (4.2)) rend parfaitement compte de ces données avec les capacités indiquées ci-dessus complétées par $\mu\left(g_{3}\right)=0.2, \mu\left(g_{1}, g_{3}\right)=0.6$. Il ne me paraît pas facile, à partir des données relatives aux six actions de référence, de rendre intelligibles les dépendances que les six valeurs de capacité retenues sont censées prendre en compte.

\section{(b) Cas d'une démarche constructive}

On suppose ici que les situations de dépendance qu'il s'agit de prendre en compte ont été concrètement décrites. Pour les raisons exposées à la fin de la Section 3.3, les situations d'auto-renforcement, d'auto-affaiblissement et d'antagonisme ne peuvent être prises en compte avec une fonction d'utilité construite en utilisant l'intégrale de Choquet. Une telle fonction est néanmoins apte à prendre en compte certaines formes de synergies positive et négative. Celles-ci font intervenir un concept d'interaction entre critères. Je me limiterai ici à l'examen du cas où ces actions ne portent que sur des paires de critères.

Les critères $g_{i}$ et $g_{j}$ sont dits indépendants si $\mu\left(g_{i}, g_{j}\right)-\left[\mu\left(g_{i}\right)+\mu\left(g_{j}\right)\right]=0$. Lorsque cette différence n'est pas nulle, c'est son signe qui discrimine synergie positive et synergie négative.

Plus précisément, la synergie positive est caractérisée ( $c f$. [14]) par le fait que 《l'importance de $g_{i}$ et $g_{j}$ pris ensemble », soit $\mu\left(g_{i}, g_{j}\right)$, est plus grande que la somme des importances individuelles $\mu\left(g_{i}\right)+\mu\left(g_{j}\right)$. Cette inégalité est toutefois difficile à interpréter. Il suffit de se reporter à l'exemple 5 ci-dessus mais, plus généralement, aux formules (4.2) et (4.3) pour percevoir que cette « importance de $g_{i}$ et $g_{j}$ pris ensemble $»$ que traduit la capacité $\mu\left(g_{i}, g_{j}\right)$ n'est pas aisément intelligible. De surcroît, « les importances individuelles de $g_{i}$ et $g_{j} »$ que traduisent respectivement $\mu\left(g_{i}\right)$ et $\mu\left(g_{j}\right)$ ne s'interprètent pas davantage de façon simple : soulignons que ces quantités diffèrent des degrés d'importance des critères concernés que modélisent les valeurs de Shapley $\phi\left(g_{i}\right)$ et $\phi\left(g_{j}\right)$. Ce n'est pas non plus la différence $\mu\left(g_{i}, g_{j}\right)-\left[\mu\left(g_{i}\right)+\mu\left(g_{j}\right)\right]$ qui reflète l'intensité de l'interaction mais un indice plus compliqué noté $I\left(g_{i}, g_{j}\right)$ (voir aussi [11]). 
Exemple 5 (suite). Si on applique aux données de cet exemple les formules qui définissent le degré d'importance (valeur de Shapley) et l'indice d'interaction, on obtient les résultats suivants :

\begin{tabular}{|l|l|l|l|}
\hline Critères & $g_{1}$ & $g_{2}$ & $g_{3}$ \\
\hline$\mu\left(g_{i}\right)$ & 0.4 & 0.5 & 0.2 \\
\hline$\phi\left(g_{i}\right)$ & 0.308 & 0.508 & 0.184 \\
\hline
\end{tabular}

\begin{tabular}{|l|l|l|l|}
\hline Critères & $\left(g_{1}, g_{2}\right)$ & $\left(g_{2}, g_{3}\right)$ & $\left(g_{1}, g_{3}\right)$ \\
\hline$\mu\left(g_{i}, g_{j}\right)-\left[\mu\left(g_{i}\right)+\mu\left(g_{j}\right)\right]$ & 0.05 & 0.20 & 0 \\
\hline$I\left(g_{i}, g_{j}\right)$ & -0.125 & 0.025 & -0.175 \\
\hline
\end{tabular}

D'après la différence $\mu\left(g_{1}, g_{2}\right)-\left[\mu\left(g_{1}\right)+\mu\left(g_{2}\right)\right]$, l'interaction est positive et, pourtant, l'indice d'interaction $I\left(g_{1}, g_{2}\right)$ est négatif. $g_{1}, g_{3}$ sont censés être indépendants puisque $\mu\left(g_{1}, g_{3}\right)-\left[\mu\left(g_{1}\right)+\mu\left(g_{3}\right)\right]=0$. Pourtant, l'intensité de l'interaction $I\left(g_{1}, g_{3}\right)$ vaut -0.175 .

Alors que $\sum_{i \in F} \mu\left(g_{i}\right)$ n'a aucune raison de valoir 1, les valeurs de Shapley sont définies de telle sorte que $\sum_{i \in F} \phi\left(g_{i}\right)=1$. Il n'en demeure pas moins que $\mu\left(g_{i}\right)$ et $\phi\left(g_{i}\right)$ reflètent l'un et l'autre un aspect de l'importance du critère $g_{i}$. Grabisch et Perny [14] parlent d'importance individuelle à propos de $\mu\left(g_{i}\right)$ et de degré d'importance à propos de $\phi\left(g_{i}\right)$. Partant de ce qui se cache sous cette différence terminologique, peut-on expliquer pourquoi $\frac{\mu\left(g_{1}\right)}{\mu\left(g_{2}\right)}=0.8$ alors que $\frac{\phi\left(g_{1}\right)}{\phi\left(g_{2}\right)}=0.6$ ?

Ces difficultés d'interprétation rendent malaisée l'utilisation de ces indices (et il en est de même pour quelques autres; $c f$. [10]) pour guider le mode de prise en compte de situations de dépendances concrètement exprimées. Il convient d'ailleurs de se demander au préalable si elles peuvent l'être avec le modèle d'agrégation que constitue l'intégrale de Choquet. Je vais examiner brièvement pour terminer le cas où les situations concrètement décrites mettent en évidence des dépendances au sens des préférences comme illustré dans l'Exemple 3. L'Exemple 5 a montré que l'intégrale de Choquet pouvait être apte à modéliser cette forme de dépendances. Toutefois, le lien qui existe entre les dépendances souhaitées et les valeurs des capacités qui les prennent en compte me paraît être fort obscur. Quoi qu'il en soit, une fois énoncé un certain nombre d'exigences que le modèle à construire doit satisfaire pour rendre compte des situations décrites, on peut :

(i) Soit trouver les $2^{n}-2$ valeurs à attribuer aux capacités pour satisfaire suffisamment bien ces exigences mais alors sera-t-on en mesure de fournir à l'interrogé des arguments lui permettant d'interpréter la signification de ces valeurs et de s'assurer qu'elles ne fabriquent pas d'artefacts gênants?

(ii) Soit prouver qu'il est impossible d'attribuer des valeurs aux capacités pour satisfaire convenablement les exigences. 
Exemple 6. Dans Grabisch and Labreuche [11], les auteurs présentent un exemple relatif à un directeur de faculté qui considère que :

- pour un étudiant «bon » en mathématiques, les langues sont plus importantes que les statistiques;

- pour un étudiant «mauvais » en mathématiques, les statistiques sont plus importantes que les langues.

La paire de critères \{langues, statistiques\} n'est donc pas indépendante au sens des préférences dans la famille des trois critères considérés. En faisant intervenir des actions de référence particulières, les auteurs ont prouvé que l'intégrale de Choquet est inapte à prendre en compte ces exigences. Cela tient au fait qu'elles font intervenir (de façon implicite) une valeur frontière entre « bon » et « mauvais ». On est ici en présence d'échelles dites bipolaires. Les auteurs présentent une généralisation de l'intégrale de Choquet (modèle avec des bi-capacités) qui permet de prendre en compte les échelles bipolaires. Le nombre des paramètres auxquels il faut attribuer une valeur s'en trouve très fortement augmenté et l'utilisation du modèle pour l'aide à la décision encore plus compliquée.

Le lecteur trouvera également dans Roy ([25] d'autres exemples qui mettent en évidence les difficultés auxquelles on se heurte avec l'intégrale de Choquet pour prendre en compte, de façon satisfaisante, d'autres types d'exigences formulées a priori.

\section{Conclusion}

Dans cet article, je me suis efforcé de montrer que, avant même de vouloir prendre en compte des dépendances entre les critères d'une famille $F$ préalablement construite, il était indispensable, dans une perspective d'aide à la décision, de commencer par chercher à clarifier la nature des dépendances qui pouvaient être en question. J'ai proposé pour cela quelques définitions et illustré, sur des exemples, des cas de dépendances dignes d'intérêt.

Dans la Section 3, j'ai tout d'abord cherché à mettre en évidence les conditions à satisfaire pour que, lorsque l'on procède par questionnement afin de recueillir des informations susceptibles de mettre en évidence la présence possible de dépendances, les réponses fournies par l'interrogé puissent être regardées comme probantes. J'ai expliqué pourquoi il pouvait être malaisé de cerner certaines formes de dépendances, ce qui m'a finalement conduit à m'intéresser à trois types de situations de dépendances à la fois simples à diagnostiquer et à faire comprendre.

$\mathrm{Au}$ début de la Section 4, j'ai rappelé que les modes d'agrégation usuels, de type analytique, supposaient que les critères de $F$ puissent être traités en faisant abstraction de toute forme de dépendances qui pourraient être présentes. J'ai signalé l'existence d'une généralisation des modèles de type ELECTRE aptes à prendre en compte les trois types de situations de dépendances définies au 3.3 et cela avec des échelles associées aux critères qui peuvent rester purement ordinales et sur lesquelles la mauvaise connaissance peut être modélisée à l'aide de seuils d'indifférence et de préférence. 
Sans en examiner en détail les limites pratiques, j'ai évoqué les possibilités qu'offrent les modèles fondés sur un système de règles pour prendre en compte des formes variées de dépendances. C'est là un sujet qui mériterait sans doute d'être approfondi.

J'ai enfin consacré toute la sous-section 4.2 à une analyse assez détaillée de l'usage qui peut être fait, dans une perspective d'aide à la décision, du modèle d'agrégation qu'offre l'intégrale de Choquet pour construire une fonction d'utilité. J'ai surtout cherché à mettre en évidence les difficultés que l'on peut rencontrer aussi bien pour construire cette fonction d'utilité que pour interpréter les formes de dépendances prises en compte dans une démarche descriptive ou encore pour modéliser, dans une démarche constructive, des situations de dépendances énoncées en termes concrets.

Remerciements. Je sais gré à Denis Bouyssou, José Figueira, Salvatore Greco et Patrice Perny d'avoir bien voulu relire attentivement une version préliminaire de cet article. Leurs remarques et suggestions fort pertinentes m'ont été précieuses pour l'améliorer. Je tiens aussi à remercier Dominique François qui, comme d'habitude, a frappé, relu et corrigé avec beaucoup de soin le présent texte.

\section{RÉFÉRENCES}

[1] S. Angilella, S. Greco, F. Lamantia and B. Matarazzo, Assessing non-additive utility for multicriteria decision aid. Eur. J. Oper. Res. 158 (2004) 734-744.

[2] A.R. Azibi, Construction de critères en aide à la décision: Aspects méthodologiques, techniques et pratiques, Université Paris-Dauphine, Thèse de doctorat en informatique (2003).

[3] A.R. Azibi and D. Vanderpooten, Construction of rule-based assignment models. Eur. J. Oper. Res. 138 (2002) 274-293.

[4] R. Bisdorff, Logical foundations of multicriteria preference aggregation, in Aiding Decisions with Multiple Criteria - Essays in Honor of Bernard Roy, edited by D. Bouyssou, E. Jacquet-Lagrèze, P. Perny, R. Slowiǹski, D. Vanderpooten and P. Vincke. Kluwer Academic Publishers (2001) 379-403.

[5] J. Figueira, S. Greco and M. Ehrgott, Multiple Criteria Decision Analysis - State of the Art Surveys. Springer Science+Business Media, Inc. (2005)

[6] J. Figueira, S. Greco and B. Roy, ELECTRE methods with interaction between criteria: An extension of the concordance index, Université Paris-Dauphine, Cahier du LAMSADE $\mathrm{N}^{\circ}$ $242(2006)$.

[7] C. Gonzales and P. Perny, GAI Networks for Decision Making under Certainty, in Proc. of the $19^{\text {th }}$ Interntional Joint Conference on Artificial Intelligence - Workshop on Advances in Preference Handling (2005) 100-105.

[8] M. Grabisch, The application of a fuzzy integral in multicriteria decision making, Eur. J. Oper. Res. 89 (1996) 445-457.

[9] M. Grabisch, Une approche constructive de la décision multicritère, Traitement du Signal 22 (4) (2005).

[10] M. Grabisch, Évaluation subjective, in Concepts et méthodes pour l'aide à la décision 3 : Analyse multicritère, edited by D. Bouyssou, D. Dubois, M. Pirlot and H. Prade. Lavoisier, Paris (2006) 179-239.

[11] M. Grabisch and C. Labreuche, Fuzzy measures and integrals in MCDA, in Multiple Criteria Decision Analysis - State of the Art Surveys, edited by J. Figueira, S. Greco and M. Ehrgott, Springer Science+Business Media, Inc. (2005) 563-608. 
[12] M. Grabisch, C. Labreuche and J.C. Vansnick, On the extension of pseudo Boolean function for the interacting bipolar criteria. Eur. J. Oper. Res. 148 (2003) 28-47.

[13] M. Grabisch, H.T. Nguyen and E.A. Walker, Fundamentals of uncertainty calculi, with applications to fuzzy inference. Kluwer Academic Publishers, Dordrecht (1995).

[14] M. Grabisch and P. Perny, Agrégation multicritère, in Logique floue, principes, aide à la décision, edited by B. Bouchon-Meunier and C. Marsala. Hermès, Paris (2003) 81-120.

[15] S. Greco, B. Matarazzo and R. Slowinski, The use of rough sets and fuzzy sets in MCDM, in Multicriteria Decision Making - Advances in MCDM Models, Algorithms, Theory, and Applications, edited by T. Gal, T.J. Stewart and T. Hanne. Kluwer Academic Publishers, Boston (1999) 14-1-14-59.

[16] S. Greco, B. Matarazzo and R. Slowinski, Conjoint measurement and rough set approach for multicriteria sorting problems in presence of ordinal criteria, in A-MCD-A - Aide Multicritère à la Décision - Multiple Criteria Decision Aiding, edited by A. Colorni, M. Paruccini and B. Roy. European Commission, Joint Research Centre, EUR Report 19808 EN (2001) 117-144.

[17] S. Greco, B. Matarazzo and R. Slowinski, Decision rule approach, in Multiple Criteria Decision Analysis - State of the Art Surveys, edited by J. Figueira, S. Greco and M. Ehrgott. Springer Science+Business Media, Inc. (2005) 507-561.

[18] R.L. Keeney and F. Raiffa, Decisions with multiple objectives: Preferences and value tradeoffs. John Wiley and Sons (1976).

[19] C. Labreuche and M. Grabisch, The Choquet integral for the aggregation of interval scales in multicriteria decision making. Fuzzy Sets and Systems 137 (2003) 11-26.

[20] H. Le Boulanger and B. Roy, L'entreprise face à la sélection des projets de recherche : La méthodologie en usage dans le groupe SEMA, in Rationalisation des choix budgétaires sous la dir d'un groupe de spécialistes animé par J. Agard, Monographies de Recherche Opérationnelle 112. Dunod, Paris (1970) 175-205.

[21] J.L. Marichal and M. Roubens, Determination of weights of interactive criteria from a reference set, Eur. J. Oper. Res. 124 (2000) 641-650.

[22] A. Rico, Modélisation des préférences pour l'aide à la décision par l'intégrale de Sugeno, thèse de doctorat, Université Paris I, France (2002).

[23] B. Roy, Méthodologie multicritère d'aide à la décision. Paris, Economica (1985).

[24] B. Roy, Meaning and validity of interactive procedures as tools for decision making. Eur. J. Oper. Res. 31 (1987) 297-303.

[25] B. Roy, Double pondération pour calculer une moyenne : Pourquoi et comment? RAIRO Oper. Res. 41 (2007) 125-139.

[26] B. Roy and D. Bouyssou, Aide multicritère à la décision : Méthodes et cas. Economica, Paris (1993).

[27] J. Stefanowski and D. Vanderpooten, A general two-stage approach to inducing rules from examples, in Rough Sets, Fuzzy Sets and Knowledge Discovery, Proc. of the International Workshop RSKD'93, Banff, Canada, edited by W. Ziarko. Springer Verlag, Berlin (1994) $317-325$.

[28] J. Stefanowski and D. Vanderpooten, Induction of decision rules in classification and discovery-oriented perspectives. Int. J. Intell. Syst. 16, 1 (2001) 13-27. 